\title{
Subdivision-Based Multilevel Methods for Large Scale Engineering Simulation of Thin Shells
}

\author{
Seth Green \\ Mechanical Engineering \\ University of Washington \\ Seattle, WA 98105; USA \\ sgreen@u.washington.edu
}

\author{
George Turkiyyah \\ Civil Engineering \\ University of Washington \\ Seattle, WA 98105; USA \\ george@ce.washington.edu
}

\author{
Duane Storti \\ Mechanical Engineering \\ University of Washington \\ Seattle, WA 98105; USA \\ storti@me.washington.edu
}

\begin{abstract}
This paper presents a multilevel algorithm to accelerate the numerical solution of thin shell finite element problems described by subdivision surfaces. Subdivision surfaces have become a widely used geometric representation for general curved three dimensional boundary models and thin shells as they provide a compact and robust framework for modeling 3D geometry. More recently, the shape functions used in the subdivision surfaces framework have been proposed as candidates for use as finite element basis functions in the analysis and simulation of the mechanical deformation of thin shell structures. When coupled with standard solvers, however, such simulations do not scale well. Run time costs associated with high-resolution simulations $\left(10^{5}\right.$ degrees of freedom or more) become prohibitive.

The main contribution of the paper is to show that the subdivision framework can be used for accelerating such simulations. Specifically the subdivision matrix is used as the intergrid information transfer operator in a multilevel preconditioner. The method described in the paper allows the practical simulation or a broad range of problems. Included examples show that the run time of the algorithm presented scales nearly linearly in time with problem size.
\end{abstract}

\section{Categories and Subject Descriptors}

J.2 [Physical Sciences and Engineering]: Engineering; J.6 [Computer-Aided Engineering]: Computer-aided de$\operatorname{sign}(\mathrm{CAD})$

\section{General Terms}

Algorithms, Design

\section{Keywords}

Thin Shell, Numerical Algorithm, Multilevel, Multiresolution, Multigrid, Subdivision Surface

Permission to make digital or hard copies of all or part of this work for personal or classroom use is granted without fee provided that copies are not made or distributed for profit or commercial advantage and that copies bear this notice and the full citation on the first page. To copy otherwise, to republish, to post on servers or to redistribute to lists, requires prior specific permission and/or a fee.

SM'02, June 17-21, 2002, Saarbrucken, Germany.

Copyright 2002 ACM 1-58113-506-8/02/0006 ...\$5.00.

\section{INTRODUCTION}

Subdivision surfaces have become widely used geometric representations of general curved three dimensional boundary models and thin shell objects. Their compactness, generality and hierarchical structure have provided effective mechanisms to support evaluating, querying, editing, fitting, and manipulating $3 \mathrm{D}$ geometry in a robust and computationally efficient manner.

More recently, the shape functions used in the subdivision surfaces framework have been proposed as candidates for use as finite element basis functions for the analysis and simulation of the mechanical deformation of thin shell structures. By construction, the subdivision shape functions provide the necessary $C^{1}$ continuity requirements (and $H^{2}$ integrability) for representing the solution of the fourth-order equilibrium equations governing the behavior of thin shells. Subdivision model descriptions also provide the rather elegant property of representing both the geometry and the physics of the deformation using the same mathematical description.

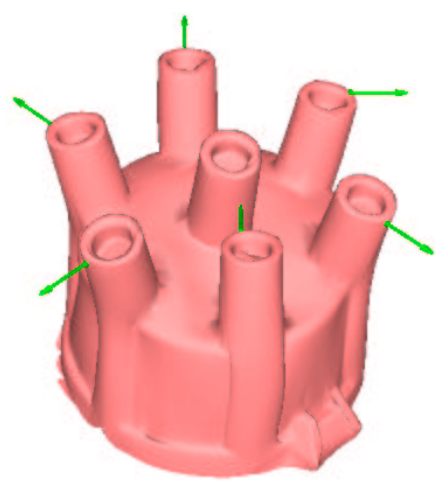

Figure 1: Distributor Cap

Figure 1 shows a simulated deformation of engineering model which is computationally expensive to compute. When coupled with standard solvers, such simulations do not scale well. Given the fourth order nature of the governing equations, the condition number of the underlying stiffness matrices grows as $O\left(\frac{1}{h^{4}}\right)$, or $O\left(n^{2}\right)$, where $h$ is a nominal element length scale and $n$ is the number of degrees of freedom in the discretized model. Using common preconditioned conjugate gradient solvers, the number of iterations grows, at best, linearly with problem size $O(n)$, and run time grows as $O\left(n^{2}\right)$ even with the most efficient sparse representations. 
In the context of high-resolution simulations involving large meshes with $n \gtrsim 10^{5}$ elements, the run time costs become prohibitive and present serious practical limits to the effective use of simulation in engineering analysis.

In this paper, we describe an algorithm that exploits the hierarchical, multilevel structure of subdivision surfaces to accelerate the convergence of solution strategies for thin shell simulations. We show that using the global subdivision matrix for inter-level propagation of information leads to solution methods in which the time to solution grows only linearly with refinement for smooth models, and provides significant acceleration for complex models with sharp features. The strategies developed are not only useful on large scale meshes but are shown to be also advantageous for relatively small problem sizes. The main contribution of the paper is to show that the subdivision framework can be used not only for representing the geometry of the solid and the mechanics of the simulation, but also for preconditioning the numerical solution. This framework allows us to construct practical simulations that are effective on a broad range of problems and problem sizes that are illustrated in the paper.

The remainder of this paper is organized as follows. We begin with a brief summary of the relevant aspects of the elements necessary to formulate the problem: subdivision surfaces, shell mechanics, and finite element discretization in sections 2, 3 and 4 respectively. Section 5 includes a discussion of multilevel solvers that leads to the presentation of our novel implementation of a multigrid-preconditioned conjugate gradient algorithm. Experimental results are presented in section 6 , and section 7 provides a discussion of the implementation aspects of our code, and the handling of sharp features.

\section{SUBDIVISION SURFACES}

Subdivision surfaces are increasingly used to represent smooth shapes for engineering design. The subdivision framework provides a compact and efficient representation for models with guaranteed smoothness properties, which are retained even if the model is deformed. Unlike traditional spline approaches, subdivision representations do not require complex cross-patch continuity constraints and can freely model objects of arbitrary topology. Recently subdivision surface representations have been shown to be effective for use in many stages of the modeling process including general modeling [10], interrogation [22], reconstruction [12, 17], shape editing [25] dynamic simulation [14] and more.
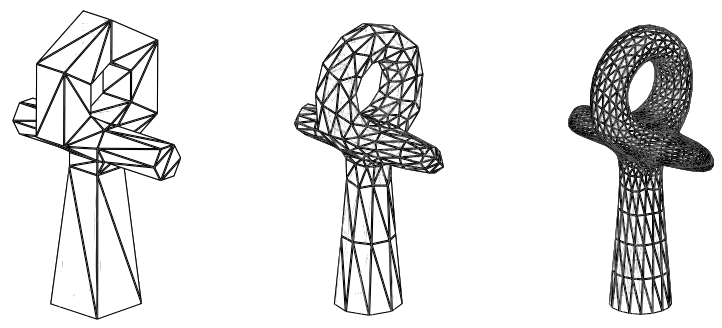

Figure 2: Subdivision Refinement (Loop's Scheme)

Subdivision surfaces are defined by an initial coarse mesh known as the control mesh. This initial mesh is refined re- peatedly by rules defined by the chosen subdivision scheme. Repeated refinements lead to a hierarchy of increasingly refined models which approach the limit surface. Figure 2 shows a model hierarchy in increasing stages of refinement. If the scheme is well formed, the refined meshes converge to a smooth surface. Most subdivision schemes are designed to produce a limit surface which smoothly approximates (but not necessarily interpolates) the initial control mesh in shape.

\subsection{Subdivision Operator}

We may refer to the vector of points representing the positions of the vertices of the mesh after $j$ levels of subdivision as $\mathbf{p}^{j}$, where $j=0$ represents the control mesh itself. The positions of the vertices at level $j$ are given by the formula

$$
\mathbf{p}^{j}=\mathbf{S}_{j-1}^{j} \mathbf{p}^{j-1}
$$

where $\mathbf{S}_{j-1}^{j}$ represents the global subdivision operator, which defines level $j$ in terms of level $j-1$, and may be represented as a rectangular (and usually sparse) matrix. The entries of $\mathbf{S}_{j-1}^{j}$ are defined by both the chosen subdivision scheme and the topology (but not geometry) of the mesh at level $j-1$.

\subsection{Loop's Scheme}

While many subdivision schemes have been proposed and analyzed, we concentrate exclusively on Loop's scheme for triangular meshes. Loop's scheme generalizes triangular box splines to meshes with arbitrary initial connectivity [13]. This scheme is not interpolating but produces surfaces which are known to be $\mathcal{C}^{2}$ continuous at all but a finite number of extraordinary points; furthermore the scheme possesses the necessary integrability [24] and refinement properties [1] to be used as a basis in thin-shell finite element analysis. Excellent reviews of Loop's subdivision scheme may be found in [21] and [7].

\section{MECHANICS OF THIN SHELLS}

A thin shell is a three dimensional body in which one geometric dimension, the thickness, is significantly smaller than the other two. In the Kirchhoff-Love thin shell framework, the deformation of the body is fully described by the deformation of its middle surface. The thin shell governing equations may be derived from kinematic, constitutive and equilibrium relations. More formal derivations may be found in [5] and [19]; here we present only the essentials of the argument.

\subsection{Kinematics}

The deformation of thin shells may be broken down into two components:

1. A straining component that occurs in the surface of the shell measuring the stretching of the surface, and hence derivable from the first fundamental form of the surface:

$$
|d s|^{2}=a_{\alpha \beta} d \theta^{\alpha} d \theta^{\beta}
$$

where $a_{\alpha \beta}=\mathbf{a}_{\alpha} \cdot \mathbf{a}_{\beta}$ is the covariant metric tensor of the surface defined by parameters $\theta^{\alpha}$ and $\theta^{\beta}$.

2. A bending component measuring the change in curvature of the shell and hence derivable from the second fundamental form of the surface

$$
B=-b_{\alpha \beta} d \theta^{\alpha} d \theta^{\beta}
$$


where $b_{\alpha \beta}=\mathbf{a}_{3} \cdot \mathbf{a}_{\beta, \alpha}$. This requires that the curvature be evaluatable at all finite regions on the manifold

If the membrane $(\boldsymbol{\epsilon})$ and bending strains $(\kappa)$ are linearized, they may be written as a linear operator in terms of displacements $(\mathbf{u})$.

$$
\left\{\begin{array}{l}
\boldsymbol{\epsilon} \\
\boldsymbol{\kappa}
\end{array}\right\}=\left[\begin{array}{l}
D_{s} \\
D_{b}
\end{array}\right] \mathbf{u}=D \mathbf{u} .
$$

\subsection{Constitutive Relations}

For linear, isotropic materials the stresses and bending moments may be written in terms of the membrane and bending strains as:

$$
\left\{\begin{array}{c}
\boldsymbol{\sigma} \\
\mathbf{m}
\end{array}\right\}=\frac{E}{1-\nu^{2}}\left[\begin{array}{cc}
h \tilde{\mathrm{C}} & 0 \\
0 & \frac{h^{3}}{12} \tilde{\mathrm{C}}
\end{array}\right]\left\{\begin{array}{l}
\boldsymbol{\epsilon} \\
\boldsymbol{\kappa}
\end{array}\right\}=C\left\{\begin{array}{l}
\boldsymbol{\epsilon} \\
\boldsymbol{\kappa}
\end{array}\right\}
$$

where $\tilde{C}$ is the standard plane stress constitutive matrix [5].

\subsection{Equilibrium Equations}

The equilibrium equations relating externally applied forces to the internal stresses (in-plane stretching/shearing forces, and bending moments) may be written through the principle of virtual work, i.e. for all admissible displacements the following relation holds:

$$
\int_{S}(\delta \boldsymbol{\epsilon}: \boldsymbol{\sigma}+\delta \kappa: \mathbf{m}) d S=\int_{S} \delta \mathbf{u} \cdot \mathbf{f} d S
$$

Substituting in the kinematics and constitutive relations of the previous sections, we arrive at the weak form of the thin shell governing equations:

$$
\int_{S} \delta D \mathbf{u}: \mathrm{C} D \mathbf{u} d S=\int_{S} \delta \mathbf{u} \cdot \mathbf{f} d S
$$

for all admissible $\delta \mathbf{u}$. Given a set of applied forces $\mathbf{f}$, we seek to determine the displacements $\mathbf{u}$ of the shell.

Kirchhoff-Love theory is the most widely used model for engineering analysis of thin shells. Other energy functionals have been studied in the contexts of dynamic simulation and reconstruction of subdivision surfaces [17].

\section{DISCRETIZATION}

The standard way of solving the governing equations is to discretize them by representing the displacements $\mathbf{u}$ as linear combinations of finite element basis functions $(N)$ with compact support [3]

$$
\mathbf{u}=N \mathbf{U}
$$

If each of the tensor quantities is "unwound" and written as a matrix Equation 7 becomes:

$$
\delta \mathbf{U}^{T}\left[\int_{S}(D N)^{T} C(D N) d S\right] \mathbf{U}=\delta \mathbf{U}^{T}\left[\int_{S} N^{T} \mathbf{f} d S\right]
$$

which can be described compactly as:

$$
\mathbf{K U}=\mathbf{F} .
$$

where $\mathbf{K}$ is the stiffness matrix and $\mathbf{F}$ is the force vector.

Cirak, et. al. $[5,4,6]$ have successfully demonstrated the utility of employing subdivision basis functions to represent both displacement fields and model geometry for thin shell finite element simulations, which requires basis functions with square integrable curvature tensors. Prior to the advent of subdivision surface bases, maintaining the required derivative continuity across patch boundaries required high order basis functions and constraints.

\subsection{Refinement}

As the number of elements (and therefore the number of basis functions) used to represent the model is increased, more accurate solutions are obtained. Figure 3 shows an example of the deflection of a curved surface modeled with increasing refinement. An upwards concentrated load has been placed near the center of the object in this simulation. When few elements are used, the local effect of the point load cannot be adequately resolved. As the mesh is subdivided more elements are added, and the numerical solution converges to the exact solution. Rates of convergence of various error estimates are described in [1] and [5].
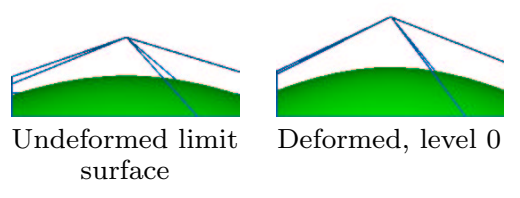

Deformed, level 0

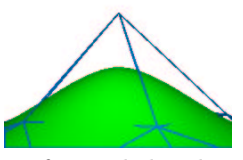

Deformed, level 1

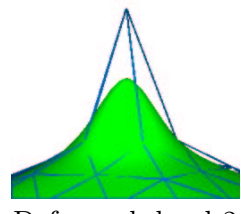

Deformed, level 2

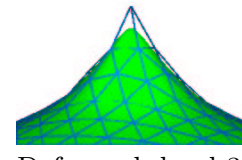

Deformed, level 3

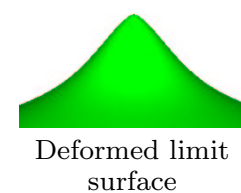

Figure 3: Refinement of Concentrated Load

Accurate solutions require a large number of elements, which is computationally expensive. A linear finite element formulation with $n$ degrees of freedom requires the inversion of a stiffness matrix $\mathbf{K}$, of size $n$ by $n$. Direct factorization methods require $O\left(n^{3}\right)$ operations to solve the system. Algorithms that take advantage of sparsity may run in $O\left(n^{2}\right)$ time [18]. Iterative methods may also be considered. As K is positive definite, the conjugate gradient algorithm (CG) is a strong candidate for a solver.

The time to solution for iterative solvers is related to the condition number of the system. The condition number in turn is related to the element size distribution in the mesh. The fourth-order nature of the equations governing the Kirchhoff thin shell model result in the condition number of the system scaling as $O\left(n^{2}\right)$. Conjugate gradient is a robust and appropriate numerical technique, but for large problems, performance $O\left(n^{2}\right)$ is unacceptable. Preconditioning the system can lower the order and drastically reduce solution effort; this is discussed in section 5.5.

\section{MULTILEVEL SOLVERS}

Multilevel solvers can mitigate the cost associated with increased accuracy by considering a hierarchy of refinements of the model instead of only a single resolution. These solution schemes accelerate the convergence of numerical sim- 
ulations by propagating solution values between finely discretized representations of a model where accurate solutions are obtained slowly, and coarsely discretized versions where approximate solutions may be obtained quickly. This process requires a hierarchical representation of multiple model resolutions with an ability to transfer information between neighboring levels (both coarse-to-fine and fine-to-coarse, denoted "T" and "R", respectively) as illustrated in Figure 4. A well designed multilevel algorithm may achieve solution times of $O(n)$.

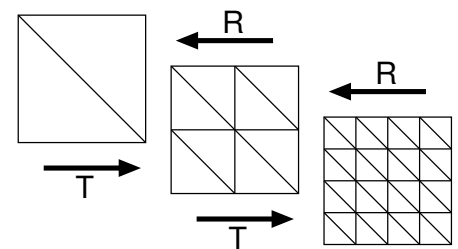

Figure 4: An Example Multilevel Hierarchy

\subsection{Multigrid}

Multigrid (MG) methods are a family of multiresolution methods that leverage the smoothing principle, which applies to many numerical solution techniques for physical systems [23]. In practice, error components with a high spatial frequency are eliminated quickly, while those with a low spatial frequency decay more slowly.

Multigrid methods leverage this smoothing property by noting that the troublesome low frequency error components on a fine discretization may be well represented on a coarser model of the problem. Two main ingredients are required: smoothing and coarse grid correction. First, an iterative scheme is used to remove high frequency error components of the solution on a finely discretized version of the problem via smoothing. Low frequency components of error may then be eliminated efficiently on a coarsely discretized model where computation is less expensive. This process may be implemented recursively over many levels. Subdivision-based finite element meshes form a natural hierarchy that can be used for successive coarse grid corrections.

\subsection{Multigrid Algorithm}

Multigrid makes use of two operations for inter-grid transfer of information. Prolongation $(T)$ and restriction $(R)$ transfer from coarse to fine and fine to coarse levels respectively. These operators are discussed in detail in sections 5.3 and 5.4. Pseudo code for the multigrid algorithm is shown below in Algorithm 1, adapted from [15], for solving the system $K u=f$. For a system with an exact solution $\hat{u}$ and an current approximate solution $u$, the residual is defined to be $r=f-K u$, the error is $e=\hat{u}-u$, and they are related as $r=K e$. Were the error known, we could compute an exact solution, but solving for the error explicitly is equivalent to solving our original problem.

The process of repeatedly refining the error at a given level is known as a $W$-Cycle. Each call to the multigrid solver that fully visits each level is known as a $V$-Cycle. Each smoothing step may require several internal iterations. The number of $\mathrm{V}$ and $\mathrm{W}-\mathrm{Cycles}$, and the number of internal smoothing iterations, have a great effect on the performance of the multigrid algorithm.

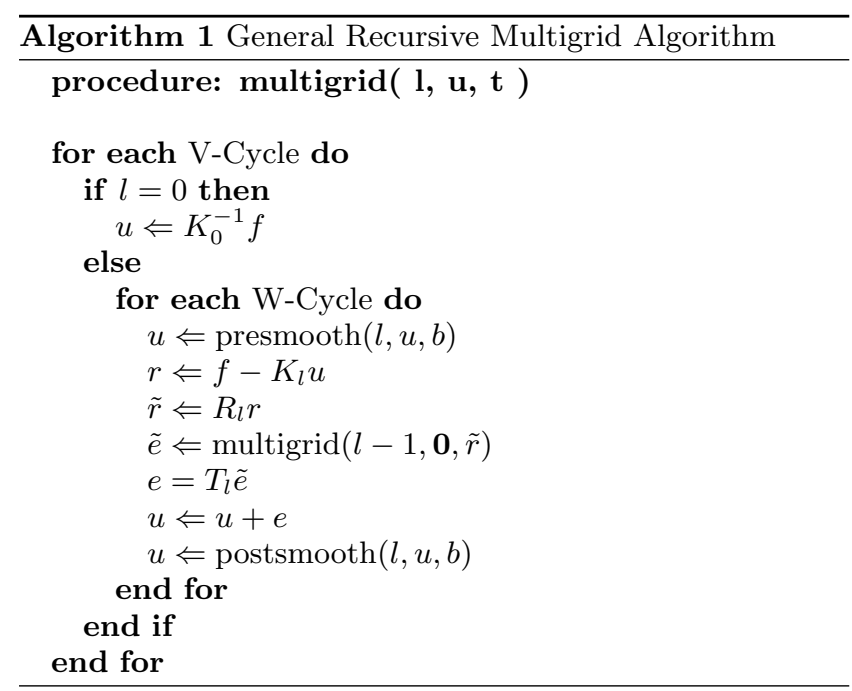

\subsection{Prolongation}

Information defined on coarse grids must be prolongated to finer levels via interpolation. Similarly, values defined on fine grids must be restricted to coarser levels, which results in a loss of information.

The choice of prolongation method is part of the design of any multigrid algorithm. We have chosen our prolongation operator between levels $j$ and $j-1$ to be the global subdivision matrix $\mathbf{S}_{\mathbf{j}-\mathbf{1}}^{\mathbf{j}}$ itself. This choice retains the intuitive feature that a prolongated limit displacement field corresponds exactly to the original limit displacement field of the coarser mesh. Thus we have now incorporated the subdivision basis in three ways into our solution scheme: it describes the geometry, the displacement, and the connection between levels in our multilevel hierarchy. The choice of prolongation operator governs the choice of restriction operator via work and energy considerations as discussed below.

\subsection{Restriction}

Physical reasoning dictates that the work done by the applied forces be equivalent for each level of the hierarchy. This energy requirement results in restriction operator being the transpose of the prolongation operator. It is well known that this transpose relation holds for interpolating basis functions generally used in finite element analysis [16] [8]. We show here that this relation holds true for the choice of the subdivision operator for prolongation, which is not interpolating in general. The work done by the generalized forces $F_{i}$ moving through generalized displacements $U_{i}$ is given by:

$$
W=\mathbf{U}^{T} \mathbf{F} .
$$

We must emphasize the use of the term generalized displacements. Unlike interpolating bases which define the nodal displacements to be the actual displacement of a point on the body at the nodal location, Loop subdivision basis functions are non-interpolating and the displacements of control mesh vertices do not possess such a simple physical interpretation. Displacements on a finer level of the mesh are given by:

$$
\mathbf{U}^{j}=\mathbf{S}_{j-1}^{j} \mathbf{U}^{j-1} .
$$

Requiring equal work on two levels for arbitrary $\mathbf{U}$ using 
the subdivision operator for restriction yields:

$$
\begin{aligned}
& \left(\mathbf{U}^{j}\right)^{T} \mathbf{F}^{j}=\left(\mathbf{U}^{j-1}\right)^{T} \mathbf{F}^{j-1} \quad \forall \mathbf{U}^{j-1} \\
& \left(\mathbf{S}_{j-1}^{j} \mathbf{U}^{j-1}\right)^{T} \mathbf{F}^{j}=\left(\mathbf{U}^{j-1}\right)^{T} \mathbf{F}^{j-1} \quad \forall \mathbf{U}^{j-1} \\
& \left(\mathbf{U}^{j-1}\right)^{T}\left(\mathbf{S}_{j-1}^{j}\right)^{T} \mathbf{F}^{j}=\left(\mathbf{U}^{j-1}\right)^{T} \mathbf{F}^{j-1} \quad \forall \mathbf{U}^{j-1} \\
& \therefore \quad\left(\mathbf{S}_{j-1}^{j}\right)^{T} \mathbf{F}^{j}=\mathbf{F}^{j-1} .
\end{aligned}
$$

This demonstrates that forces may be transferred from fine to coarse levels using the transpose of the subdivision operator. Both the prolongation and restriction operators require only a single sparse matrix vector multiply and are computationally inexpensive. Qin has proposed an interlevel transfer operator outside of the multigrid context that uses the psuedoinverse of the subdivision matrix [17].

\subsection{Multigrid Preconditioned Conjugate Gradient}

The multigrid method provides a useful framework for solving problems hierarchically, yet achieving optimal performance can be difficult. The algorithm relies on a handful of tuning parameters, including the number of $\mathrm{V}$ and $\mathrm{W}$ cycles, and the number and kind of smoothing steps to apply per iteration. Proper tuning of these parameters remains difficult, and optimal performance of the multigrid by itself can be difficult to realize in practice.

Fortunately multigrid concepts can be easily integrated into other strategies for solving linear systems. Multigrid has been shown to be significantly more effective when used to precondition the conjugate gradient algorithm (CG). CG is specifically designed to take advantage of the positive definiteness of the systems encountered in finite element simulations. Multigrid preconditioned conjugate gradient strategies (MGPCG) have been explored in mechanics and other applications $[8,15,2]$.

MGPCG combines both multigrid and conjugate gradient by using multigrid to compute the effect of a preconditioner. Multigrid may be used to solve the linear system $\mathbf{U}=\mathbf{K}^{-\mathbf{1}} \mathbf{F}$; this is exactly the computational form needed for a preconditioner. A preconditioner need not be exact though, and a full multigrid solution is expensive. Instead of running multigrid to convergence, a few $\mathrm{V}$ cycle steps will solve $\mathbf{U} \approx \mathbf{K}^{-1} \mathbf{F}$. In practice a single $\mathrm{V}$ cycle is sufficient to effectively precondition the system. The matrix $\mathbf{K}^{-\mathbf{1}}$ is never explicitly formed, only its effect on vectors is needed.

\section{RESULTS}

We have run a variety of examples to verify and test the performance of our method. These examples include a distributor cap and fan housing (courtesy Hugues Hoppe), a square flat plate, a hollow coiled tube, and a model with non-trivial genus inspired by the ancient Egyptian "Ankh" symbol. Each of these models and their simulations are shown in figure 5 (the fan housing is shown separately in figure 10). The left column of figure 5 shows the coarsest level control meshes used to define the models, the middle column shows sample loading conditions used for testing, and the right column shows the calculated deformed shape. The two rightmost columns are rendered after two levels of subdivision each.
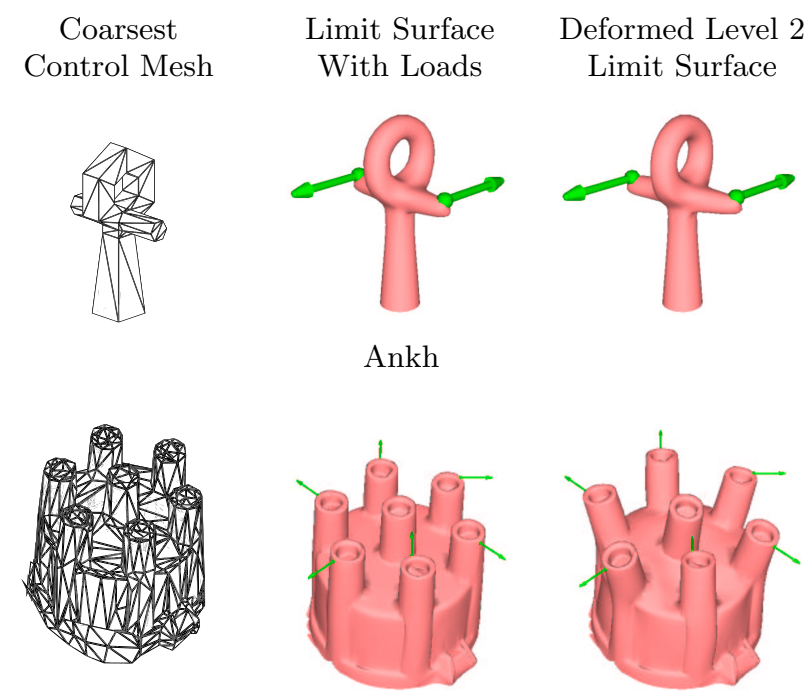

Distributor Cap
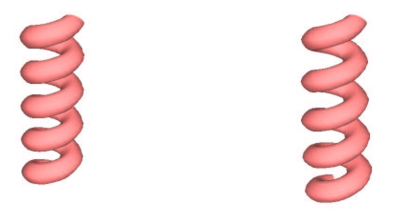

Coil
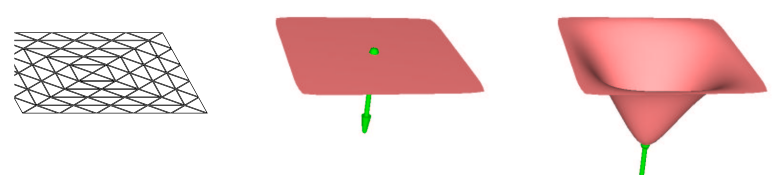

Plate

Figure 5: Models

For comparison, we have tested our own MGPCG solver against two other solution techniques, unpreconditioned conjugate gradient and SuperLU [9]. Unpreconditioned CG was selected to compare scaling behavior and contrast the effectiveness of using a multigrid preconditioner in our solver. SuperLU is an efficient general sparse direct matrix solver that is freely available for academic use. Both MGPCG and $\mathrm{CG}$ were run until the solution residual was reduced by a factor of $10^{5}\left(r_{n} / r_{0}<10^{-5}\right)$.

Each level of subdivision increases the number of elements and degrees of freedom of the model four-fold (modulo boundary effects). Because of this, setup times for the entire model hierarchy are not significantly larger than for the finest level alone. Each model was simulated for a few different levels of subdivision refinement. The MGPCG solver always used the coarsest level for a base mesh and the number of total levels in the hierarchy was equal to the given level of subdivision. Logarithmic plots of solution time versus the number of model degrees of freedom is shown for selected experiments to demonstrate efficiency and scaling behavior; an algorithm of order $O\left(n^{p}\right)$ will show a slope of $p$ on a log-log plot. A full listing of all results is given in table 1 at the end of this section. For the simplest models, 
the very finest discretizations may be considered overkill; the experiments were performed to best show the scaling behavior of the technique for very large problem sizes.

\subsection{Plate Models}

While flat plate models may be of limited geometric interest, flat plates remain of significance in engineering analysis [8] and provide test cases where results can be compared to known analytical solutions. Figure 5 shows a flat plate simulated with a central concentrated load. We have also simulated plates with distributed loads and inhomogeneous material properties; in this last case the elastic modulus of one quarter of the plate was increased by a factor of 10 . Figures 6 and 7 plot the time to solution for various levels of subdivision refinement for the concentrated loading and inhomogeneous cases respectively.

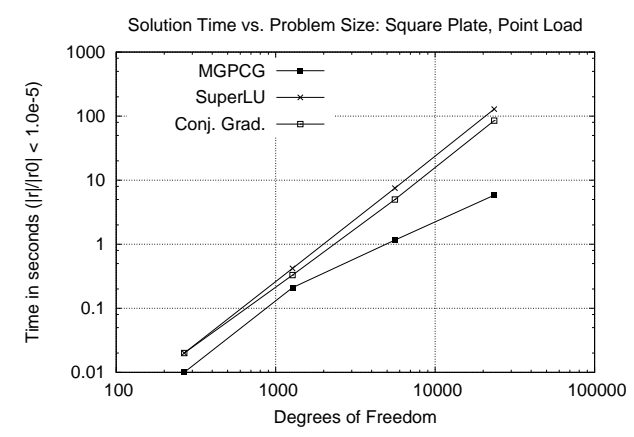

Figure 6: Homogeneous Plate, Concentrated Load

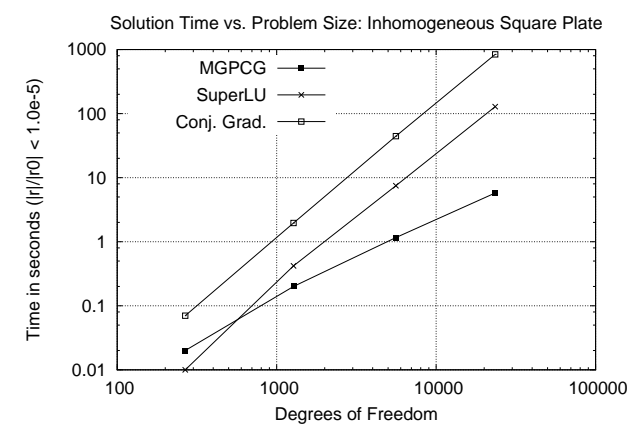

Figure 7: Inhomogeneous Plate, Distributed Load

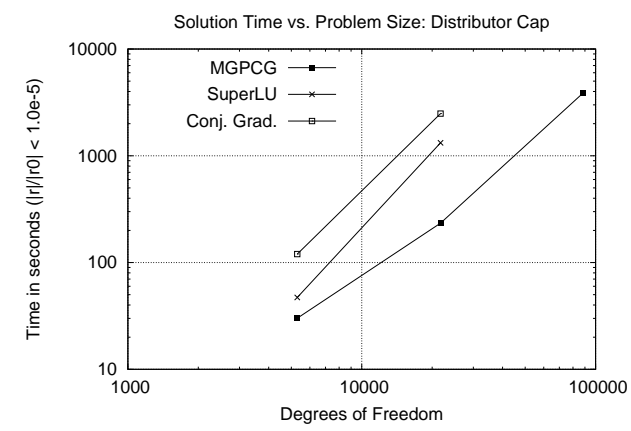

Figure 8: Distributor Cap Solution Times
Each of these plots demonstrate the superior scaling behavior of our MGPCG solution strategy. In both examples, MGPCG scales at nearly $O(n)$ while SuperLU and CG scale at nearly $O\left(n^{2}\right)$. There is little difference between the plots for the homogeneous square plate under either concentrated or distributed loading (not shown), indicating that it is only the system stiffness matrix, not the load vector which governs solver performance. While CG and SuperLU show nearly identical scaling rates in each case, the material inhomogeneity of the latter experiment greatly increases the condition number of the system and adds a large constant factor to the CG solution time. This trend is not seen with MGPCG, showing its effectiveness. Being a direct solver, SuperLU is completely unaffected by conditioning.

\subsection{Distributor Cap}

The distributor cap is the most complex model tested. The coarsest level mesh has approximately 500 vertices, 1000 faces, and 1500 edges, several of which are sharp. An enlarged image of the deformed distributor cap is shown in figure 1. Solution times are again plotted versus levels of model refinement in figure 8. Here MGPCG shows a marked speedup at all levels of refinement. Scaling improvement is not as pronounced with this simulation. Sharp features make the hierarchy more approximate than for smooth models. The discretization also contains a number of very high aspect ratio elements which contribute to ill-conditioning. The smooth coil and ankh models showed very favorable scaling and speedup results similar to the plates. The fan model also gained signifcant speedup from MGPCG.

\begin{tabular}{|c|c|c|c|c|c|}
\hline \multirow{2}{*}{ Model } & \multirow{2}{*}{$\begin{array}{l}\text { Subdi- } \\
\text { visions }\end{array}$} & \multirow{2}{*}{ DOF } & \multicolumn{3}{|c|}{ Time } \\
\hline & & & MGPCG & SLU & CG \\
\hline \multirow{5}{*}{ Coiled Tube } & & 336 & & $0.06 \mathrm{sec}$ & $0.59 \mathrm{sec}$ \\
\hline & 1 & 1344 & $0.45 \mathrm{sec}$ & $0.69 \mathrm{sec}$ & $8.66 \mathrm{sec}$ \\
\hline & 2 & 5376 & $2.59 \mathrm{sec}$ & $18.05 \mathrm{sec}$ & $1.27 \mathrm{~min}$ \\
\hline & 3 & 21504 & $11.01 \mathrm{sec}$ & $11.07 \mathrm{~min}$ & $9.76 \mathrm{~min}$ \\
\hline & 4 & 86016 & $50.67 \mathrm{sec}$ & $43.46 \mathrm{~min}$ & $2.46 \mathrm{hr}$ \\
\hline \multirow{5}{*}{$\begin{array}{l}\text { Square Plate, } \\
\text { Distributed } \\
\text { Load }\end{array}$} & 0 & 51 & & $0.01 \mathrm{sec}$ & $<.01 \mathrm{sec}$ \\
\hline & 1 & 267 & $0.02 \mathrm{sec}$ & $0.02 \mathrm{sec}$ & $0.03 \mathrm{sec}$ \\
\hline & 2 & 1275 & $0.22 \mathrm{sec}$ & $0.43 \mathrm{sec}$ & $0.31 \mathrm{sec}$ \\
\hline & 3 & 5595 & $1.19 \mathrm{sec}$ & $7.51 \mathrm{sec}$ & $5.14 \mathrm{sec}$ \\
\hline & 4 & 23451 & $5.77 \mathrm{sec}$ & $2.14 \mathrm{~min}$ & $1.41 \mathrm{~min}$ \\
\hline \multirow{4}{*}{$\begin{array}{l}\text { Distributor } \\
\text { Cap }\end{array}$} & 0 & 1269 & & $1.97 \mathrm{sec}$ & $3.01 \mathrm{sec}$ \\
\hline & 1 & 5301 & $30.12 \mathrm{sec}$ & $47.13 \mathrm{sec}$ & $2.00 \mathrm{~min}$ \\
\hline & 2 & 21717 & $3.90 \mathrm{~min}$ & $22.04 \mathrm{~min}$ & $41.42 \mathrm{~min}$ \\
\hline & 3 & 88029 & $1.07 \mathrm{hr}$ & & \\
\hline \multirow{4}{*}{ Fan Disk } & 0 & 378 & & $0.08 \mathrm{sec}$ & $0.68 \mathrm{sec}$ \\
\hline & 1 & 1302 & $3.28 \mathrm{sec}$ & $1.62 \mathrm{sec}$ & $8.12 \mathrm{sec}$ \\
\hline & 2 & 4803 & $18.87 \mathrm{sec}$ & $31.63 \mathrm{sec}$ & $1.86 \mathrm{~min}$ \\
\hline & 3 & 18393 & $3.07 \mathrm{~min}$ & $14.25 \mathrm{~min}$ & \\
\hline \multirow{5}{*}{$\begin{array}{l}\text { Inhomogeneous } \\
\text { Square Plate }\end{array}$} & 0 & 51 & & $<.01 \mathrm{sec}$ & $<.01 \mathrm{sec}$ \\
\hline & 1 & 267 & $0.02 \mathrm{sec}$ & $0.01 \mathrm{sec}$ & $0.07 \mathrm{sec}$ \\
\hline & 2 & 1275 & $0.20 \mathrm{sec}$ & $0.42 \mathrm{sec}$ & $1.96 \mathrm{sec}$ \\
\hline & 3 & 5595 & $1.16 \mathrm{sec}$ & $7.49 \mathrm{sec}$ & $44.52 \mathrm{sec}$ \\
\hline & 4 & 23451 & $5.76 \mathrm{sec}$ & $2.14 \mathrm{~min}$ & $14.01 \mathrm{~min}$ \\
\hline \multirow{6}{*}{ Ankh } & 0 & 174 & & $0.02 \mathrm{sec}$ & $0.18 \mathrm{sec}$ \\
\hline & 1 & 693 & $0.41 \mathrm{sec}$ & $0.25 \mathrm{sec}$ & $2.43 \mathrm{sec}$ \\
\hline & 2 & 2757 & $2.50 \mathrm{sec}$ & $4.60 \mathrm{sec}$ & $19.89 \mathrm{sec}$ \\
\hline & 3 & 10989 & $11.69 \mathrm{sec}$ & $1.18 \mathrm{~min}$ & $2.43 \mathrm{~min}$ \\
\hline & 4 & 43869 & $45.69 \mathrm{sec}$ & $33.87 \mathrm{~min}$ & . \\
\hline & 5 & 175293 & $2.77 \mathrm{~min}$ & & \\
\hline \multirow{5}{*}{$\begin{array}{l}\text { Square Plate, } \\
\text { Point Load }\end{array}$} & 0 & 51 & & $0.01 \mathrm{sec}$ & $<.01 \mathrm{sec}$ \\
\hline & 1 & 267 & $0.01 \mathrm{sec}$ & $0.02 \mathrm{sec}$ & $0.02 \mathrm{sec}$ \\
\hline & 2 & 1275 & $0.21 \mathrm{sec}$ & $0.42 \mathrm{sec}$ & $0.33 \mathrm{sec}$ \\
\hline & 3 & 5595 & $1.16 \mathrm{sec}$ & $7.50 \mathrm{sec}$ & $4.99 \mathrm{sec}$ \\
\hline & 4 & 23451 & $5.84 \mathrm{sec}$ & $2.15 \mathrm{~min}$ & $1.42 \mathrm{~min}$ \\
\hline
\end{tabular}

Table 1: Timing Results

\subsection{Notes}

SuperLU is optimized for very high performance; our MGPCG implementation, while optimal algorithmically, has not been tuned to this level and a reimplementation would likely result in significant constant factor speed increase. Being a direct solver, SuperLU also can consume very large amounts of memory during execution (two or more gigabytes for large problems) and was not used for the very largest simulations. 
Timings were performed on a $1.2 \mathrm{GHz}$ Athlon CPU with 1.5 Gb of RAM. Missing entries in Table 1 are due to lack of memory for SuperLU or patience for large simulations when MGPCG clearly outperforms other methods. MGPCG requires at least two levels to form a hierarchy, thus no results are listed for the coarsest discretization in the MGPCG column.

\section{IMPLEMENTATION}

\subsection{Data Structures}

We have created a hierarchical variant of the quad-edge structure originally described by Guibas and Stolfi [11]. The quad-edge is a powerful structure for storing boundary manifolds of arbitrary topology. It allows constant time access to all immediate neighbors of a vertex, face, or edge by storing both the manifold edge graph and its dual. We have modified the structure to allow for the explicit storage of subdivision hierarchies by adding a parent and child field to each directed edge. Each non-boundary directed edge is related to four children, and each child is assigned a single parent.
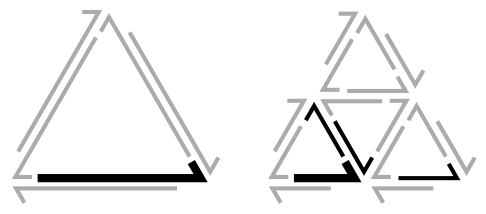

Figure 9: Hierarchy Representation

The child edge that shares the same origin and direction as its parent is denoted the primary child, and all other child edges may be derived from it in constant time, thus requiring only one child pointer. Figure 9 shows a triangle of a mesh, formed of half-edges, before and after one step of subdivision; the parent edge and the primary child are indicated in extra-bold, the non primary children in bold. The convention for the primary/non-primary children was chosen because it extends directly to modeling quadrilateral subdivision schemes.

This hierarchy scheme is useful for generating the global subdivision operator $\mathbf{S}_{\mathbf{j}-\mathbf{1}}^{\mathbf{j}}$ in constant time per vertex. Traditionally, the global subdivision not explicitly formed for producing subdivision geometry as it is only applied once [7]. However, we find it advantageous to represent this operator in a sparse matrix data structure as it is applied to the model each step of MGPCG.

\subsection{Sharp Features}

Sharp features may be represented in the subdivision framework by modifying the subdivision rules along the edge of a sharp crease [12]; unfortunately infinitely sharp edges violate the strict curvature continuity requirements of thin shell theory, and are difficult to evaluate analytically. DeRose et. al. [10] introduced the idea of semi-sharp creases which allow selectively sharp corners to be modeled. Semi sharp edges may be created by applying a finite number of sharp subdivision steps, then reverting to the smooth rules thereafter. In our implementation sharp rules are used when subdividing the mesh, and mechanical properties are evaluated using smooth basis functions at the current level; thus a model's sharpness is determined by the current level of subdivision. Like the geometric representation itself, sharp features are refined by subdivision.

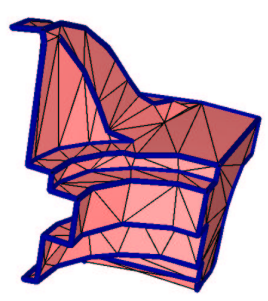

Control mesh

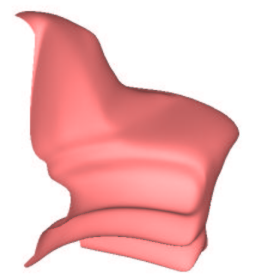

Smooth limit surface

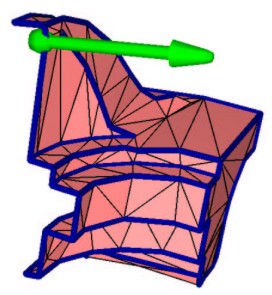

Model with applied load

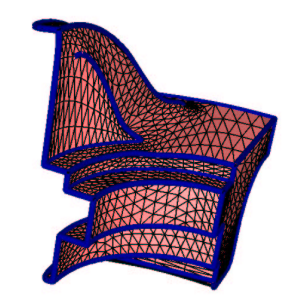

2 Sharp subdivisions

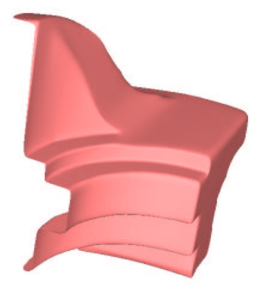

Smooth limit surface after 2 sharp subdivisions

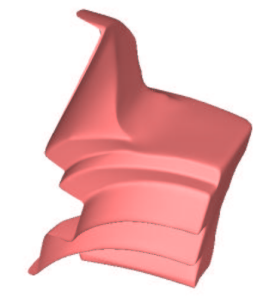

Deformed limit surface
Figure 10: Representation and Deformation of a $\mathrm{Hi}$ erarchical Fan Housing Model

Two levels of an example hierarchy for an engineering model of a fan housing are demonstrated in figure 10. The left column shows the coarsest level and the right column shows the model after two levels of subdivision. The topleft image shows the coarsest level control mesh with sharp edges marked, the top-right image shows the same model after two levels of subdivision. The middle-left image shows the very smooth limit surface derived from the coarse mesh with sharp features suppressed; this is the geometry which is defined by the coarsest model. The middle-right image shows the smooth limit surface that is generated after two levels of subdivision using sharp rules; the model still has smooth, finite curvature everywhere, but has much more distinct features. The bottom-left image shows the coarse model loading conditions; the arrow represents a concentrated load applied to the tip of the model. The bottomright image shows the model after deformation. The computation was done on the model with two levels of subdivision. Simulating the coarsest level does not provide accurate results as the control mesh has too few degrees of freedom, and the very smooth limit surface generated (middle-left) does not model the design features of the model well. However it can capture the gross motion of the body well and that information is leveraged at finer levels in the hierarchy. 


\subsection{Evaluation at Arbitrary Parameter Values}

In areas of regular connectivity (where all vertices have six neighbors), Loop's scheme reduces by design to a limit surface defined by the triangular box spline polynomials [13]. Any points lying within a regular region may be determined directly through evaluation of the box spline basis functions without recourse to subdivision. Those areas of the mesh which do not possess regular connectivity are called extraordinary, and are more difficult to evaluate.

Extraordinary regions shrink to a point in the limit, so each non-extraordinary point will eventually lie in a regular region after some number of subdivision steps. Stam [20] [21] has demonstrated that the eigenstructure of the local subdivision matrix may be exploited to evaluate the subdivision basis functions and their derivatives at any point on the mesh in constant time. Stam analyzed faces with one extraordinary point only, but by considering the basis functions on a finer level to be linear combinations of the level above, the technique may be applied to arbitrary coarse meshes with no connectivity restrictions. Forming the stiffness matrix requires integrating equation 9. All integration is performed by numerical quadrature; the basis functions are evaluated using Stam's technique.

\section{CONCLUSIONS}

We have shown that a multigrid-preconditioned conjugate gradient solver can efficiently solve thin shell mechanics problems defined by subdivision geometry, including sharp features. Our solver exploits the natural hierarchy of a subdivision model to solve smooth problems in near $O(n)$ time and to provide significant speedup for a variety of thin shell simulations. Subdivision approaches for thin shell modeling have previously been shown to be both an elegant and robust description for models of arbitrary topology. Here we have shown the subdivision operator to be an effective description for inter-level transfer of information in a hierarchical model. Specifically we use the global subdivision matrix and its transpose as the restriction and prolongation operators in a multigrid-preconditioned conjugate gradient solver implementation. Subdivision surface basis functions now form a triumvirate of representation techniques for thin shell simulation: they serve to define the model's geometry, deformation and precondition the numerical solution.

\section{ACKNOWLEDGEMENTS}

This work was supported by NFS's ITR program, grant \#ACI-0086093. The author also thanks Cole Brooking for help in creating models.

\section{REFERENCES}

[1] G. Arden. Approximation Properties of Subdivision Surfaces. PhD thesis, University of Washington, 2001.

[2] S. Ashby and R. Falgout. A Parallel Multigrid Preconditioned Conjugate Gradient Algorithm for Groundwater Flow Simulations. Nuclear Science and Engineering, (124):145-159, 1996.

[3] Bathe. Finite Element Procedures. Prentice-Hall, Englewood Clffs, N.J., 1996.

[4] F. Cirak and M. Ortiz. Fully $C^{1}$-conforming subdivision elements for finite deformation thin-shell analysis. International Journal for Numerical Methods in Engineering, 51(7):813-833, July 2001.
[5] F. Cirak, M. Ortiz, and P. Schröder. Subdivision Surfaces: a New Paradigm for Thin-Shell Finite-Element Analysis. International Journal for Numerical Methods in Engineering, 47(12):2039-72, April 2000.

[6] F. Cirak, M. J. Scott, E. Antonsson, M. Ortiz, and P. Schröder. Integrated Modeling, Finite-Element Analysis, and Engineering Design for Thin-Shell Structures using Subdivision Surfaces. Preprint.

[7] P. Schröder D. Zorin, editor. SIGGRAPH: Subdivision Course Notes, CDROM supplement, 2000.

[8] W. G. Davids and G. M. Turkiyyah. Multigrid Preconditioner for Unstructured Nonlinear 3D FE Models. Journal of Engineering Mechanics, 125(2):186-196, February 1999.

[9] J. W. Demmel, S. C. Eisenstat, J. R. Gilbert, X. S. Li, and Joseph W. H. Liu. A supernodal approach to sparse partial pivoting. SIAM Journal on Matrix Analysis and Applications, 20(3):720-755, 1999.

[10] T. DeRose, M. Kass, and Tien Truong. Subdivision Surfaces in Character Animation. In Computer Graphics (Siggraph 1998 Proceedings), pages 85-94, 1998.

[11] L. Guibas and J. Stolfi. Primitives for the Manipulation of General Subdivisions and the Compuation of Voronoi Diagrams. ACM Transactions on Graphics, 4(2):74-123, 1985.

[12] H. Hoppe, T. DeRose, T. Duchamp, M. Halstead, H. Jin, J. McDonald, J. Schweitzer, and W. Stuetzle. Piecewise smooth surface reconstruction. Computer Graphics, 28(Annual Conference Series):295-302, 1994.

[13] C. Loop. Smooth Subdivision Surfaces Based on Triangles. Master's thesis, University of Utah, 1987.

[14] C. Mandal, H. Qin, and B. C. Vemuri. A novel fem=based dynamic framework for subdivision surfaces. In Sixth ACM Symposium on Solid Modeling and Applications. ACM Press, 1999.

[15] J. C. Meza and R. S. Tuminaro. A Multigrid Preconditioner for the Semiconductor Equations. SIAM J Sci. Comput., 17(1):118-132, January 1996.

[16] I. D. Parsons and J. F. Hall. The Multigrid Method in Solid Mechanics: Part I - Algorithm Description and Behavior. International Journal for Numerical Methods in Engineering, 29:719-737, 1990.

[17] Hong Qin, Chhandomay Mandal, and Baba C. Vemuri. Dynamic catmull-clark subdivision surfaces. IEEE Transactions on Visualization and Computer Graphics, 4(3):215-229, 1998.

[18] Y. Saad. Iterative Methods for Sparse Linear Systems. PWS Publishing Company, Boston, MA, 1996.

[19] J. C. Simo and D. D. Fox. On A Stress Resultant Geometrically Exact Shell Model. Part I: Formulation and Optimal Parameterization. Computer Methods in Applied Mechanics and Engineering, 72:267-304, 1989.

[20] J. Stam. Exact Evaluation of Catmull-Clark Subdivision Surfaces at Arbitrary Parameter Values. In Computer Graphics, pages 395-404. ACM, 1998.

[21] J. Stam. Exact Evaluation of Loop Triangular Subdivision Surfaces at Arbitrary Parameter Values. In Computer Graphics. ACM, 1998. CD-ROM Supplement.

[22] G. Taubin. Is This A Quadrisected Mesh? In D. C. Anderson and K. Lee, editors, Sixth ACM Symposium on Solid Modeling and Applications, pages 261-266. ACM Press, 2001.

[23] U. Trottenberg, C. Oosterlee, and A. Schüller. Multigrid. Academic Press, London, UK, 2001.

[24] P. Schröder U. Reif. Curvature integrability of subdivision surfaces. Advances in Computational Mathematics, 14(2):157-174, 2001.

[25] D. Zorin. Stationary Subdivision and Multiresolution Surface Representation. PhD thesis, Cal.Tech., 1998. 